\title{
SOME RESULTS OF HIGH-SPEED TWO-CHANNEL \\ PHOTOMETRY OF WOLF-RAYET STARS WITH SUSPECTED COMPACT COMPANIONS
}

\author{
B.E. ZHILYAEV, V.R. KHALACK and I.A. VERLYUK \\ Main Astronomical Observatory, Kiev, AS Ukraine
}

\begin{abstract}
Strong transient chaotic high-frequency activity has been detected in WR148 on a time scale of a few minutes. The main property is the variability of the fluctuation power, not the light-curve. The same is true for WR136 but with a more permanent pattern. The power of fluctuations is of order of thousandth relative to the steady intensity. The maximum fluctuation power lies in the frequency band of $25-50 \mathrm{~Hz}$.
\end{abstract}

Key words: stars: Wolf-Rayet - flickering - neutron stars - black holes

\section{Introduction}

A compact companion orbiting in the WR wind should produce short-lived effects caused by accretion on a time-scale of milliseconds. In order to find these, high time-resolution photometry is needed. An extensive search for short-term variability of WRs summarized by Marchenko et al. (1994) failed to give the desired results. A new energetic test for detection of small-scale chaotic variability was developed by the authors. A transient high-frequency flickering was detected in two WR stars with suspected compact companions.

\section{Observations}

Monitoring of the two most likely candidates among WR stars with possible compact companions, WR136 (WN6+c?) and WR148 (WN7+BH?), was carried out. The observations were obtained at the $60 \mathrm{~cm}$ telescope at the Mount Terskol Observational Station in the North Caucasus $(3100 \mathrm{~m})$. using a high-speed two-channel photometer (Zhilyaev et al. 1992). Simultaneous recording of a reference star was performed for accurate scintillation power measurements during all the observations. High-time-resolution monitoring was done in $U B V$ and narrow-band filters centered on the continuum at $4270 \AA$ and on the He II $4686 \AA$ emission line. Integration times in the range $4 \mathrm{~ms}$ to $1 \mathrm{sec}$ were applied.

\section{An algorithm for detection of chaotic variability}

Denote the sampling values of the mean count-rate and of the variance as $\overline{\mathrm{n}}$ and $s^{2}$, respectively. The variance has the physical meaning of the power 
of fluctuations. It consists of three components, caused by Poisson noise, scintillations and intrinsic stellar variability:

$$
s^{2}=s_{p}^{2}+s_{s c}^{2}+s_{\star}^{2}
$$

The first term is equal to the mean count rate of star and background.

$$
s_{p}^{2}=\tilde{n}=\tilde{n}_{\star}+\tilde{n}_{b}
$$

The second term measures the contribution of atmospheric scintillations to total variance. It may be defined as

$$
s_{s c}^{2}=\frac{1}{T} \int_{0}^{T}\left[f(t) n_{\star}(t)\right]^{2} d t=\varepsilon \tilde{n}_{\star}^{2}
$$

where $f(t)$ is the light-wave envelope in a turbulent atmosphere. The quantity $\varepsilon$ defined as

$$
\varepsilon=\frac{s^{2}-\tilde{n}}{\tilde{n}_{\star}^{2}}
$$

specifies the power of scintillations in the operating frequency range $1 / T-$ $-1 / 2 \Delta t$, where $\Delta t$ is a sampling time. The quantity $\varepsilon$ depends on atmospheric conditions as well as on the aperture of telescope. It is independent of the intensity $\tilde{n}_{\star}$ (Tatarsky 1967). Using the standard procedure (Kendall $\&$ Stuart 1946), the error variance for $\varepsilon$ can be found

$$
D[\varepsilon]=\frac{1}{\tilde{n}^{4}}\left\{D\left[s^{2}\right]+\left(\frac{2 s^{2}}{\tilde{n}}-1\right)^{2} D[\tilde{n}]\right\}
$$

where $\mathrm{D}$ is the variance operator. For variances of $\tilde{n}$ and $s^{2}$ we have

$$
D[\tilde{n}]=\frac{s^{2}}{N}, \quad D\left[s^{2}\right]=\frac{2 s^{4}}{N-1}
$$

where $\mathrm{N}$ is the length of measurement series. The actual value of $\varepsilon$ caused by scintillations can be determined from reference star data. To detect atmospheric scintillations, one can assume the null hypothesis: $s^{2}=\tilde{n}$. In view of (5) a detection threshold can be set

$$
\varepsilon_{t h}=\alpha \sqrt{D[\varepsilon]}=\frac{\alpha}{\tilde{n}} \sqrt{\frac{2}{N}}
$$

For a time series with normal density $\alpha=1.96$ corresponds to $95 \%$ confidence level. The quantity $\varepsilon$ specifies an accuracy limit of stellar photometry in the operating frequency range at given atmospheric conditions. The above quoted expressions open the way for detecting of intrinsic chaotic fluctuations of stars masked by atmospheric noise. 


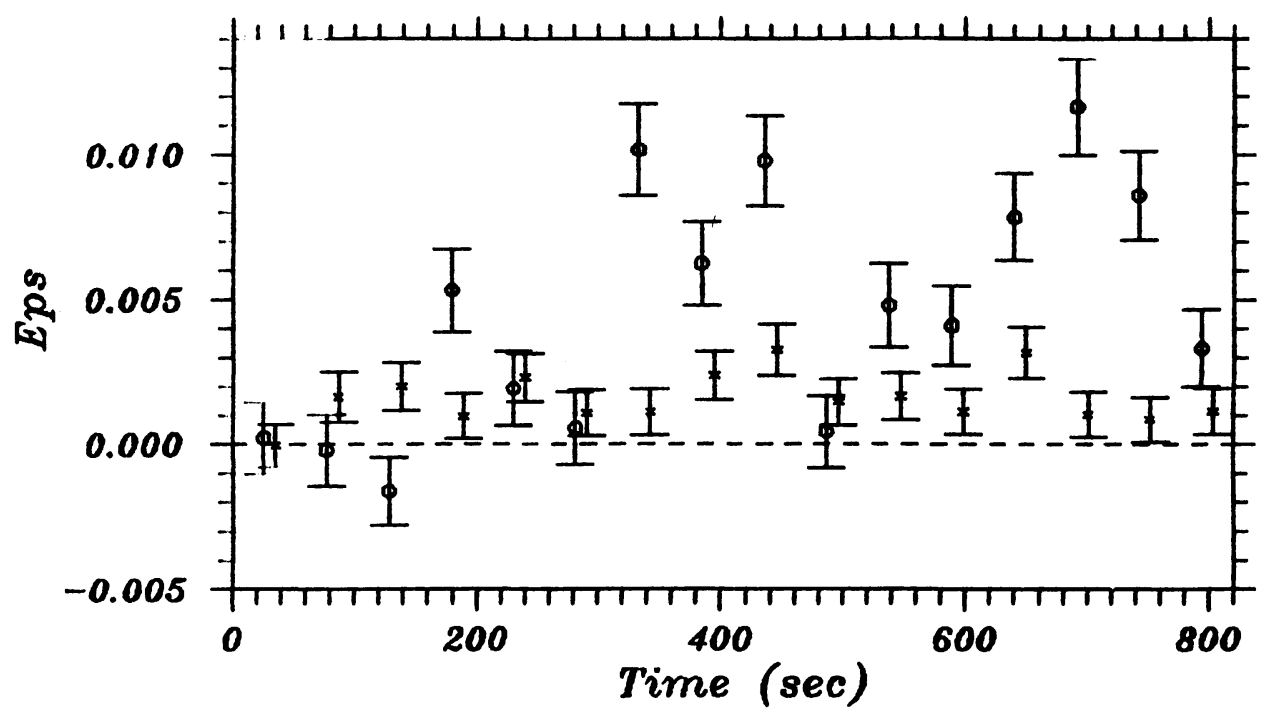

Fig. 1. Variations in the fluctuation powers $\varepsilon$ in the continuum at $4270 \AA$ of the WN7 star WR148 (top) and reference star (bottom). Each dot is a result of $51.2 \mathrm{~s}$ estimating of $\varepsilon$. Observations were carried out on 1991 October 14 with integration time $0.1 \mathrm{~s}$.

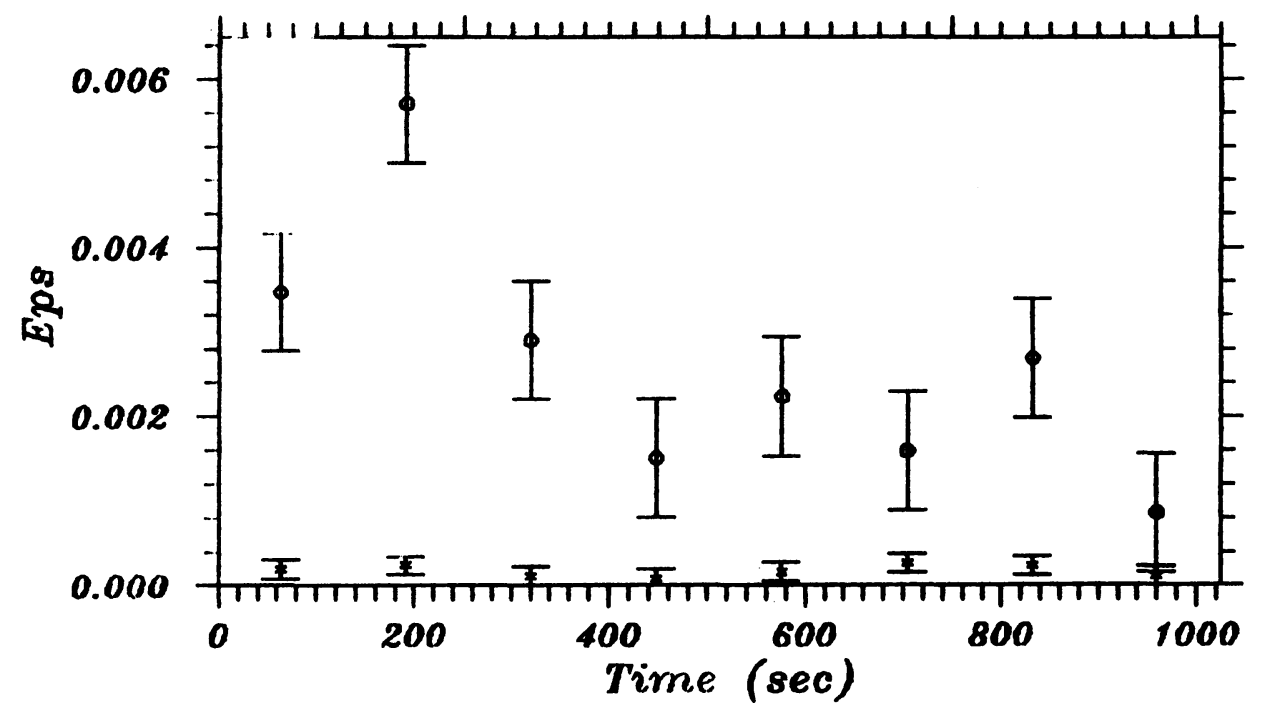

Fig. 2. The same as in Fig. 1. $128 \mathrm{~s}$ estimating of $\varepsilon$ was used on October 23, 1993, with integration time $0.25 \mathrm{~s}$. 


\section{Results}

One clearly sees from Figs. 1 and 2 that the scintillation power of a reference star is constant on average. However, strong 'flaring' activity in WR148 takes place on both occasions. The main property of this consists in variability of the fluctuation power not the light-curve. Both curves lack any regular pattern and exhibit a transient variability on a time scale of a few minutes. We can deduce from the data of WR148: (1) the light-curves show no presence of remarkable peculiarities such as flare events or coherent oscillations; (2) the maximum fluctuation power reaches 0.5 percent of the steady intensity. The power in the continuum at $4270 \AA(\Delta \lambda=110 \AA)$ is, on average, a factor of $5-10$ higher than that in $B$ band; and (3) the frequency band from 1 to $5 \mathrm{~Hz}$ contributes about $80 \%$ of the total fluctuation power. Low count-rates of WR148 set an upper limit on a value of frequency bound. In the case of WR136 the frequency interval can be extended up to $50 \mathrm{~Hz}$. The same features WR136 demonstrates in its behaviour. The maximum fluctuation power lies in the frequency band of 25 to $50 \mathrm{~Hz}$. Activity has a more permanent pattern and has been detected from time to time only.

\section{Acknowledgements}

V.K. is grateful to the Local Organizing Committee and the American Astronomical Society for financial support.

\section{References}

Kendall, M.A., Stuart, A. 1946. The Advanced Theory of Statistics, 1, (London: Griffin) Marchenko, S., Antokhin, I., Bertrand, J.-F., Lamontagne, R., Moffat, A.F.J., Piceno, A., Matthews, J. 1994, $A J$ submitted

Tatarsky, V.I. 1967, Propogation of Waves in Turbulent Atmosphere, (Moscow: Nauka)

Zhilyaev, B.E., Romaniuk, Ya.O., Svyatogorov, O.A. 1992, Sov. Astron. 69, 895 